\title{
Mitochondrial disease
}

\author{
Replacing the powerhouse of the cell
}

Mallory Frederick

\begin{abstract}
The mitochondria are called the powerhouses of the cell because they make most of the energy required by our cells. Diseases caused by mitochondrial mutations always occur in the children of women with mitochondrial disease and, due to this central role in energy production, can be extremely detrimental. A recently developed preventative therapy, mitochondrial replacement therapy, reduces carryover of mitochondrial disease from mother to child. Though these "three-parent babies" are born without mitochondrial disease, this type of gene editing carries ethical implications and unexplored effect on future generations.
\end{abstract}

\section{INTRODUCTION}

Mitochondria are often referred to as the powerhouse of the cell due to their invaluable role in cellular energy production. The 16 kilobase-pair mitochondrial genome (mtDNA) contains 37 genes, 13 of which are directly involved in energy production via oxidative phosphorylation. ${ }^{1}$ The varying energy needs of different cells types require the number of mitochondria to vary with them: any given cell contains between 100 to 1000 mitochondria, with each mitochondrion containing between two to ten copies of its mtDNA. ${ }^{2}$ The mitochondria's alkaline interior environment causes a high mutation rate in mtDNA relative to nuclear DNA. ${ }^{3}$ Additionally, given the key role of mitochondria in energy production, mtDNA mutations are often linked to disease. Such diseases, collectively referred to as mitochondrial disease, present with a wide array of symptoms. mtDNA diseases may onset as early as pregnancy or much later in life. This group of diseases includes Leigh syndrome, a neurodegenerative disease present from childhood; chronic progressive external ophthalmoplegia (CPEO), which leads to impaired vision; and a variety of neuropathies, myopathies, and encephalopathies. ${ }^{2}$ In general, the severity of the disease depends on the location and type of mutation and is reflected in the age of onset: the later a disease presents, the less severe it tends to be.

Increased knowledge of mitochondrial diseases has led to the development of multiple diagnosis strategies. While traditional histological techniques can be used to diagnose specific diseases, such as when a familial link is known, newer technologies are making diagnosis easier and faster. ${ }^{4}$ Various PCR techniques make it easy to identify point mutations, deletions, rearrangements, and in some cases mutational load. ${ }^{5}$ Conversely, treatment options remain limited and few prevention strategies exist. One recently developed prevention strategy, mitochondrial replacement therapy (MRT), relies on non-Mendelian inheritance of mitochondria during fertilization to prevent mitochondrial disease in the next generation. ${ }^{6}$ The general goal of MRT is reduction of mutated mtDNA by replacing the maternal mitochondria with donor mitochondria. This results in the child, who is often called a threeparent baby, not inheriting the mother's mitochondrial disease. Here, I provide a brief overview of the main MRT methods along with a discussion of the ethical considerations and regulatory processes associated with this new technique.

\section{MITOCHONDRIAL REPLACEMENT THERAPY TECHNIQUES}

One of the earliest methods proposed to reduce inheritance of mutated mtDNA was co-injection of donor cytoplasm during in vitro fertilization (IVF), where the addition of healthy mitochondria was proposed to compensate for the naturally inherited mutant mtDNA. ${ }^{6}$ However, this method is now considered largely unsuitable due to the large volumes of donor cytoplasm required to out-compete the pre-existing mtDNA. Though no longer used, the principle of the cytoplasmic transfer method remains the basis of more advanced methods, which continue to rely on replacement of the mutant mtDNA with healthy mitochondria from a donor. ${ }^{6}$ The main difference between the two methods discussed here, maternal spindle transfer (MST) and pronuclear transfer (PNT), is the timing of fertilization during the procedure.

MST uses mature metaphase II oocytes from the mother and a donor egg, with the uniform distribution of mitochondria in oocytes minimizing carryover of mutant mtDNA.,8 The mother's nuclear DNA is extracted from the egg as a karyoplast, containing the intact nucleus and a small amount of cytoplasm enclosed in a membrane. The enucleated donor oocyte (cytoplast) is fused with the karyoplast, the resulting reconstructed oocyte is fertilized with the father's sperm, and the embryo, which contains mtDNA from the donor and nuclear DNA from the mother and father, is implanted similarly to embryos used in routine IVF procedures. Embryos produced by MST overwhelmingly contain donor mtDNA, often with less than $1 \%$ of the mtDNA derived from the mother. ${ }^{6,10}$

While MST uses unfertilized oocytes, PNT uses zygotes from the mother and the donor, with both zygotes formed using the father's genetic material. The benefit of this technique comes from the presence of two easily visualized pronuclei - one each from the sperm and oocyte - immediately before mitosis. ${ }^{8}$ The pronuclei are removed from the mother's zygote as a karyoplast and the donor zygote is enucleated. However, when fusion between the karyoplast and cytoplast occurs in PNT, mtDNA carryover from the mother occurs at a much higher rate than in MST. The clustering of mitochondria around the pronuclei at the zygote stage results in virtually all blastocysts containing mtDNA from both the 
mother and the donor. ${ }^{8}$ With some optimization of the procedure, researchers have reduced the amount of mtDNA carryover to less than $2 \%{ }^{6,10}$ Though PNT is therefore not as effective as MST, each strategy has different advantages and areas for further development.

\section{ETHICAL CONSIDERATIONS \& REGULATIONS}

As with any newly developed technology, the ethical implications of creating three-parent babies through MRT must be considered. Firstly, the safety of MST, PNT, and other therapeutic technologies should be evaluated early in the development process. The United Kingdom opened the first clinic to perform MRT in 2016 with the stipulation that all participants must be involved in long-term studies, potentially over generations, to understand the full effects of the technology. ${ }^{6}$ As MRT access continues to grow, the number of potential participants will improve our understanding of the mechanisms involved in mitochondrial disease.

In addition to safety concerns, parental rights should be considered. In most procedures that rely on egg donors, the egg donor relinquishes their rights to any children. MRT is different from other strategies that rely on egg donors, however, as the child's complete genome is derived from three separate sources: the mother, the father, and the egg donor. The situation is further complicated in lesbian relationships. Sourcing the cytoplast from one mother and the karyoplast from the other would create a child with genetic material from both parents, but if the egg donor does not have rights to the child, the cytoplast mother is not considered a parent in the same manner as the karyoplast mother. This broadens concerns regarding MRT access, and whether it should be limited only to those whose children would be directly impacted by mitochondrial disease without MRT.

Concerns regarding extended access are also linked to concerns that too much freedom will eventually result in abuse of these types of technologies leading to creation of "designer babies" with unnecessary genetic modifications, such as specific hair or eye colours. With the advent of precision molecular techniques such as the CRISPR/Cas9 system making genome modification easier than ever, it is essential that the line not be blurred between what types of modifications are and are not ethically permissible. Thus, it is generally agreed that access should continue to be limited based on necessity in order to prevent abuse of these technologies. ${ }^{6,8,10}$

MRT access is limited by governmental regulation in most countries. The only country where MRT is currently legal is the United Kingdom, while in Canada and the United States, MRT is banned on the basis of being a heritable genetic modification. ${ }^{11}$ As of 2018, the governments of Australia and Singapore are considering amendments to allow MRT treatments to proceed. In general, countries are wary of MRT legalization due to the risks intrinsic to genetic modification-replacing the mitochondria may create a slippery slope to modification or replacement of the whole nuclear genome. ${ }^{12}$ Conversely, the specification that MRT only be used when inheritance of mitochondrial disease is certain maintains the integrity of the field and is often not regarded as genetic editing comparable to precision molecular editing technologies, which may have increased potential for abuse.

\section{CONCLUSION}

Mitochondrial diseases present as a spectrum of disorders that can range in severity over a person's life. When mitochondrial diseases are encoded within maternal germline mtDNA, inheritance is certain. However, new technologies are making mtDNA replacement easy and efficient, effectively eliminating the risk of inheritance. ${ }^{6,8,10}$ The low rate of mtDNA carryover makes MRT an appealing option for potential parents. While currently an expensive procedure, MRT has the potential to eliminate mitochondrial disease from the maternal line, an investment for the future. As with any new technology, governmental regulations of MRT are currently very restrictive. ${ }^{11,12}$ Despite this, MRT as a cure for mitochondrial disease is a growing field, with new methods being developed even as older methods are improved. ${ }^{6,8}$ As these technologies improve, it is likely that more governments will reduce regulations to allow this potentially life-saving procedure, accelerating the speed of development and access to cures.

\section{REFERENCES}

1. Hatefi Y. The mitochondrial electron transport and oxidative phosphorylation system. Annu Rev Biochem. 1985 Jul;54(1):1015-69. https://doi.org/10.1146/annurev.bi.54.070185.005055

2. Tuppen HA, Blakely EL, Turnbull DM, et al. Mitochondrial DNA mutations and human disease. Biochim Biophys Acta. 2010 Feb;1797(2):113-28. https://doi.org/10.1016/j.bbabio.2009.09.005

3. Starkov AA. The role of mitochondria in reactive oxygen species metabolism and signaling. Ann NY Acad Sci. 2008 Dec;1147(1):37-52. https://doi.org/10.1196/annals.1427.015

4. Taylor RW, Schaefer AM, Barron MJ, et al. The diagnosis of mitochondrial muscle disease. Neuromuscular Disord. 2004 Apr;14(4):237-45. https://doi.org/10.1016/j.nmd.2003.12.004

5. Taylor RW, Taylor GA, Durham SE, et al. The determination of complete human mitochondrial DNA sequences in single cells: Implications for the study of somatic mitochondrial DNA point mutations. Nucleic Acids Res. 2001 Aug;29(15):e74. https://doi. org/10.1093/nar/29.15.e74

6. Tachibana M, Kuno T, Yaegashi N. Mitochondrial replacement therapy and assisted reproductive technology: A paradigm shift toward treatment of genetic diseases in gametes or in early embryos. Reprod Med Biol. 2018 Sep;17(4):421-33. https://doi.org/10.1002/rmb2.12230

7. Tachibana M, Sparman M, Sritanaudomchai H, et al. Mitochondrial gene replacement in primate offspring and embryonic stem cells. Nature. 2009 Sep;461(7262):367-72. https://doi.org/10.1038/ nature 08368

8. Wolf DP, Mitalipov N, Mitalipov S. Mitochondrial replacement therapy in reproductive medicine. Trends Mol Med. 2015 Feb;21(2):68-76. https://doi.org/10.1016/j.molmed.2014.12.001

9. Sathananthan AH, Trounson AO. Mitochondrial morphology during preimplantational human embryogenesis. J Hum Reprod Sci. 2000 Jul;15(Suppl 2):148-59. https://doi.org/10.1093/humrep/15.suppl_2.148

10. Saxena N, Taneja N, Shome P, et al. Mitochondrial donation: a boon or curse for the treatment of incurable mitochondrial diseases. $\mathrm{J}$ Hum Reprod Sci. 2018 Mar;11(1):3-9. https://doi.org/10.4103/jhrs. JHRS_54_17 


\section{FEATURE ARTICLE}

11. Castro RJ. Mitochondrial replacement therapy: the UK and US regulatory landscapes. J Law and Biosciences. Dec 2016;3(3):726-35. https://doi.org/10.1093/jlb/lsw051

12. Pompei M, Pompei FJ. Overcoming bioethical, legal, and hereditary barriers to mitochondrial replacement therapy in the USA. Assist Reprod Genet. Mar 2019;36(3):383-93. https://doi.org/10.1007/s10815018-1370-7 